\title{
Complications of Laparoscopy in Connection with Entry Techniques
}

\author{
Ibrahim Alkatout, MD, PhD, MA
}

\begin{abstract}
The anatomy of the human being has not changed. However, technical developments in operating materials and methods call for improvements in surgical procedures as well as the management of complications. A fundamental distinction between any operating method and laparoscopy is that, in the latter, the initial entry is usually performed in blind fashion. Blind entry may result in vessel or organ damage, especially in patients who have undergone previous surgery. One of the difficulties associated with the entry is that the damage may not be identified immediately and then necessitate major abdominal repair. Furthermore, the improvement of surgical instruments and techniques enables the surgeon to perform even major operations by the laparoscopic approach. This is associated with renewed learning curves and a high rate of complications due to vascular, bowel, uterine, or bladder damage. The improvement of surgical techniques must be accompanied by advancements in the management of complications. The aim of this review is to address the risks of laparoscopy as well as their correct and professional management. (J GYNECOL SURG 33:81)
\end{abstract}

Keywords: laparoscopy, entry technique, complication, management, bowel injury, bladder injury

\section{Introduction}

$\mathbf{T}$ HE ANATOMY OF THE HUMAN BEING has not changed. However, technical developments in operating materials and methods call for improvements in surgical procedures as well as the management of complications. A fundamental distinction between any operating method and laparoscopy is that, for the latter, the initial entry is usually performed in a blind fashion. Blind entry may result in vessel or organ damage, especially in patients who have undergone previous surgery. One of the difficulties associated with the entry is that the damage might not be identified immediately and then could necessitate major abdominal repair. Furthermore, the improvement of surgical instruments and techniques enables the surgeon to perform even major operations using the laparoscopic approach. This is associated with renewed learning curves and a high rate of complications due to vascular, bowel, uterine, or bladder damage. The improvement of surgical techniques must be accompanied by advancements in the management of complications.

\section{Counseling and Informed Consent}

Patients undergoing laparoscopic procedures must be informed of the risks and potential complications as well as alternative surgical methods. Counseling before laparoscopy should include a discussion of the entry technique used and the risks associated with laparoscopic entry ${ }^{1,2}$ : injury to the bowel; the urinary tract; blood vessels; omentum; and other surrounding organs. Potential complications at a later point in time include wound infections, pain due to adhesions, and the formation of hernias.

The counseling must include the patient's individual risk depending on his/her body mass index, potential obesity, or significant underweight, as well as any immunosuppression that may be present. Depending on the patient's medical history, it would be important to take anatomical malformations, midline abdominal incisions, and/or a history of peritonitis or inflammatory bowel disease into account. ${ }^{3,4}$

\section{Trocar Placement}

Elevation of the abdominal wall by means of a sufficient pneumoperitoneum is a precondition for safe trocar insertion. Excepted from this rule is blind entry without a pneumoperitoneum. An insufflation pressure up to $25 \mathrm{~mm}$ $\mathrm{Hg}$ can be tolerated by the patient. The optic trocar may be placed indirectly $(10 \mathrm{~mm})$ or in two steps, inserting a $5-\mathrm{mm}$ trocar first and then dilating to $10 \mathrm{~mm}$ in case the surgeon

Department of Gynecology and Obstetrics, Kiel School of Gynecological Endoscopy, University Hospitals Schleswig-Holstein, Kiel, Germany.

(C) Ibrahim Alkatout 2017; Published by Mary Ann Liebert, Inc. This is an Open Access article distributed under the terms of the Creative Commons Attribution License, which permits unrestricted use, distribution, and reproduction in any medium, provided the original work is properly cited. 

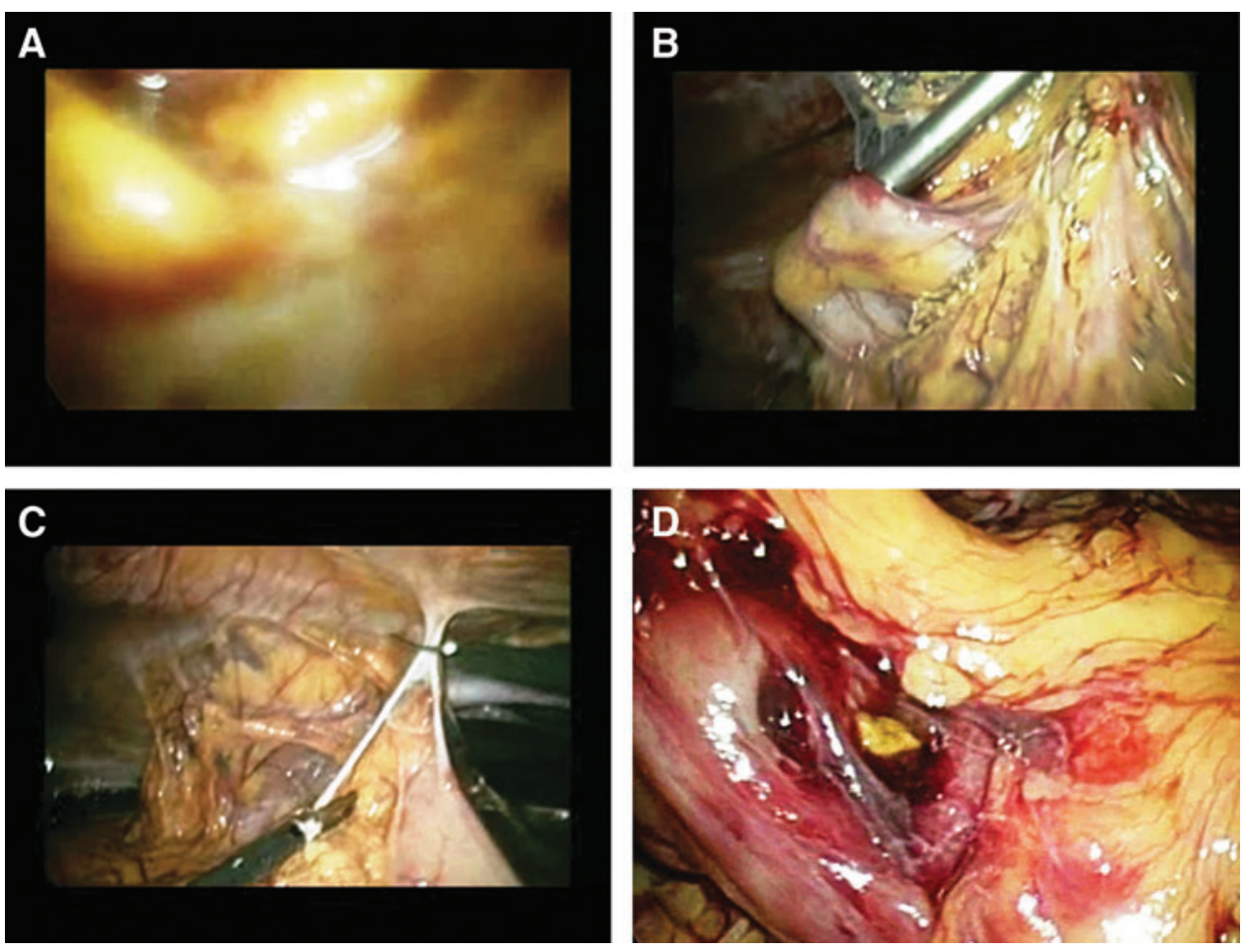

FIG. 1. (A) Entering the primary trocar site with a 5 -mm optical trocar; stool is visible in the distant parts of the trocar. (B) The injury is clearly seen after inserting an ancillary trocar in the lower abdomen. It is important to leave the primary trocar in its respective location. (C) After mobilizing the adhesions that fixed the bowel to the abdominal wall, (D) the defect can be demarcated and sutured. anticipates any bleeding, intra-abdominal abnormality, or adherent bowel loops. If the surgeon suspects adherence of the bowel in the umbilical region, the primary trocar site must be visualized from a secondary port site, such as the lower abdominal wall, with a 5 -mm laparoscope (Fig. 1). ${ }^{3}$

All ancillary trocars must be inserted with an intraabdominal pressure of $20-25 \mathrm{~mm} \mathrm{Hg}$ under direct vision in order to achieve the maximum distance between the abdominal wall and the inner organs. The inferior epigastric vessels are visualized laparoscopically, whereas the superficial vessels can be visualized by diaphanoscopy. Thus, it is ensured that the entry points are at a distance from the respective vessels (Figs. 2 and 3). The number of ancillary trocars may vary, but all of them must be in- serted under direct viewing. For safe insertion, the two major superficial vessels in this region (i.e., the superficial epigastric artery and the superficial circumflex iliac artery) must be avoided. ${ }^{3,5-7}$

For a patient with a significantly high risk of adhesions, a history of abdominal surgery including caesarian section, a large fibroid uterus, an umbilical hernia, large ovarian cysts, preperitoneal gas insufflation, or failed umbilical entry, Palmer described in 1974 an abdominal entry point in the midclavicular line, $\sim 3 \mathrm{~cm}$ below the costal margin. ${ }^{6} \mathrm{Pal}-$ mer's point can be used for the Veress needle as well as for small trocars. In patients with scars or previous operations in the upper left quadrant, the Lee-Huang point is an alternative (Fig. 4).
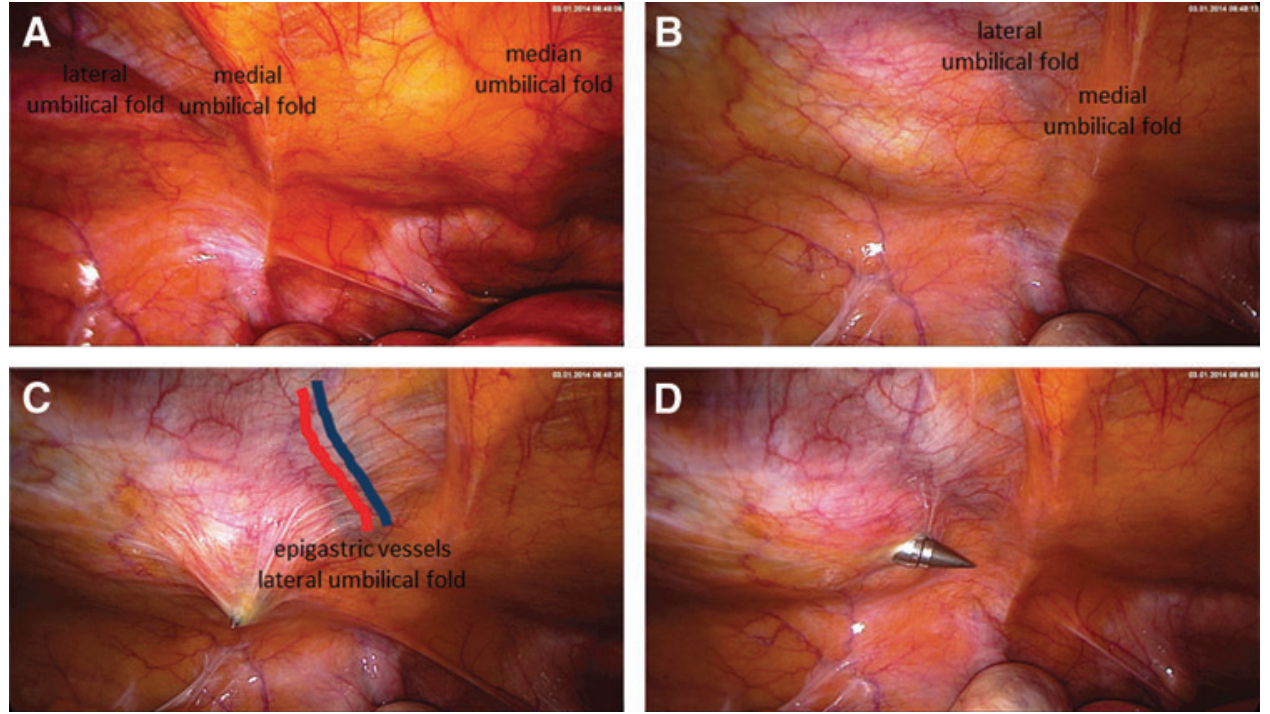

FIG. 2. Secondary trocar placement, left lower abdominal entry. (A) The three plicae are visualized. (B) The palpating finger is showing the area lateral to the lateral umbilical fold. (C) Entry of the sharp ancillary trocar lateral to the lateral umbilical fold. (D) Once the peritoneum has been penetrated, the trocar heads toward the fundus of the uterus and thus avoids injury to the major vessels and the bowel. 
FIG. 3. (A) Acute bleeding from the inferior epigastric artery. (B and $\mathbf{C}$ ) The surgeon's view is obliterated within seconds. Blood flows at the site; the situation is obviously precarious. (B-D) The artery is closed by the inside-out suturing technique and the suture is fixed to the abdomen.
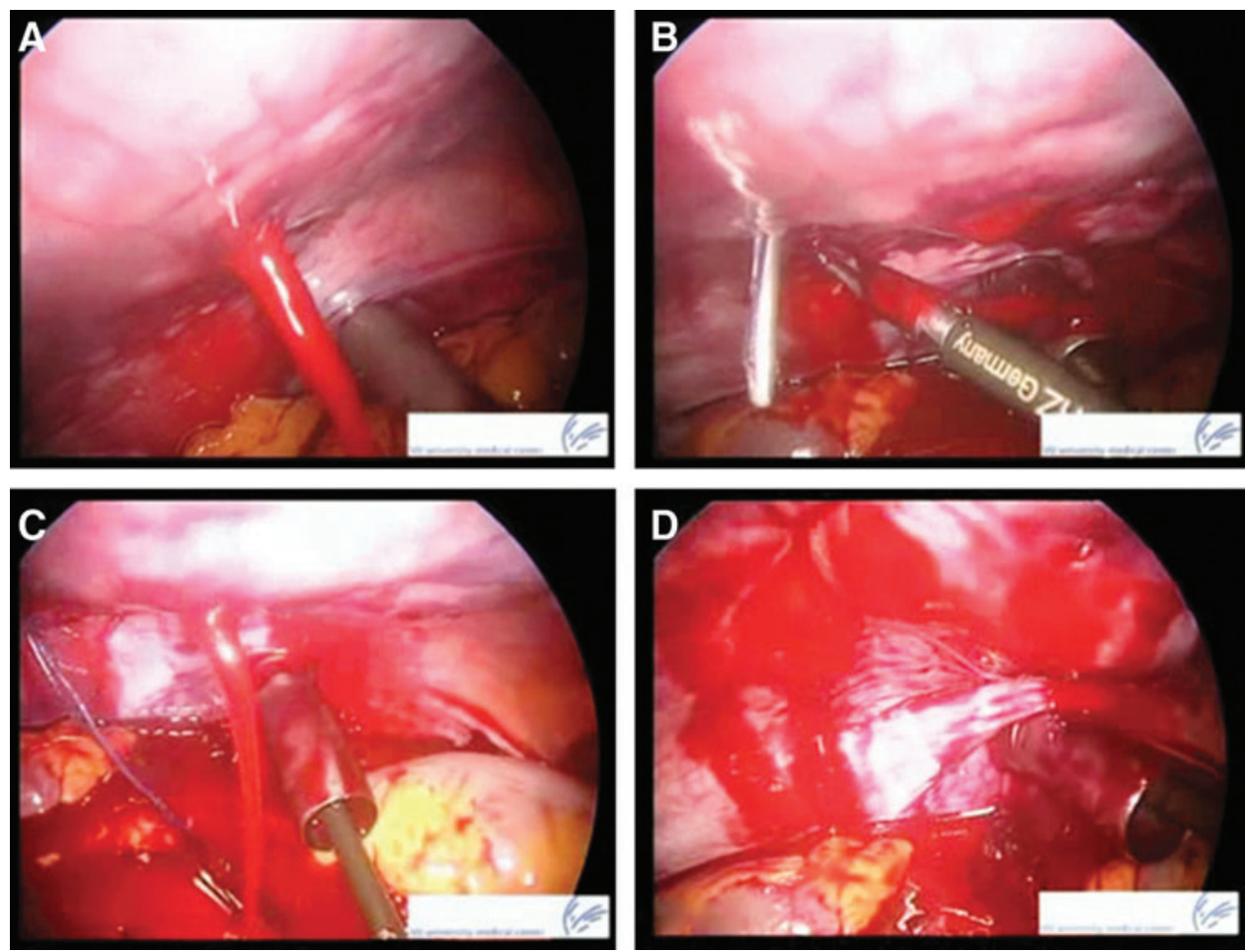

Indications for the use of Palmer's point are the following:

- After two failed attempts to achieve subumbilical insufflation

- Very obese patients

- Patients who have undergone previous abdominal surgery and/or those with suspected intra-abdominal adhesions

- Patients with previous longitudinal abdominal incisions.

\section{Complications in Port Placement}

Complications of laparoscopy can be divided into early complications, which are recognized immediately and can be resolved instantly, and delayed or late complications that occur after a time interval in the postoperative period. The latter are more difficult to manage because the patients will have been discharged from the hospital or the symptoms may be unspecific. ${ }^{8,9}$
FIG. 4. (A) The LeeHuang point can be palpated directly beneath the xiphoid. (B-D) Alternative entry site showing Palmer's point. It is situated in the midclavicular line, about $3 \mathrm{~cm}$ below the costal margin, and is used in patients with known or anticipated umbilical adhesions.
A
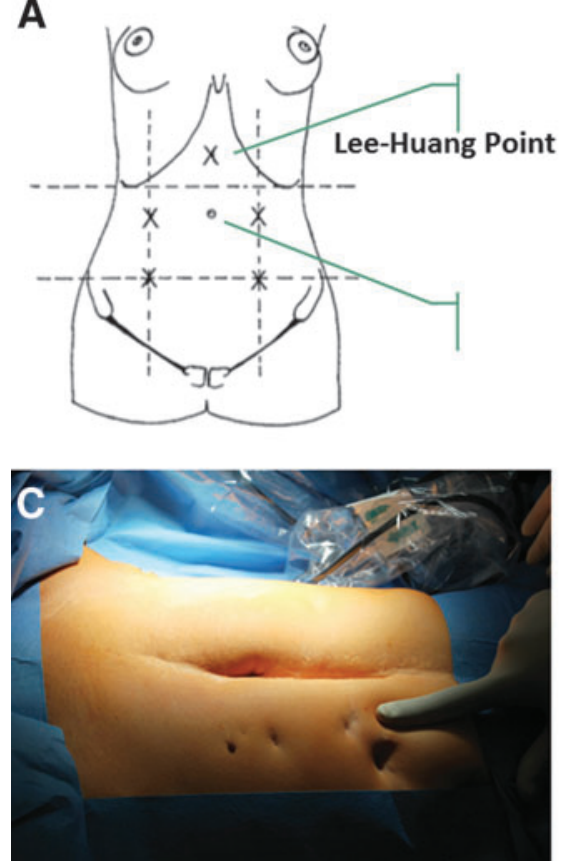
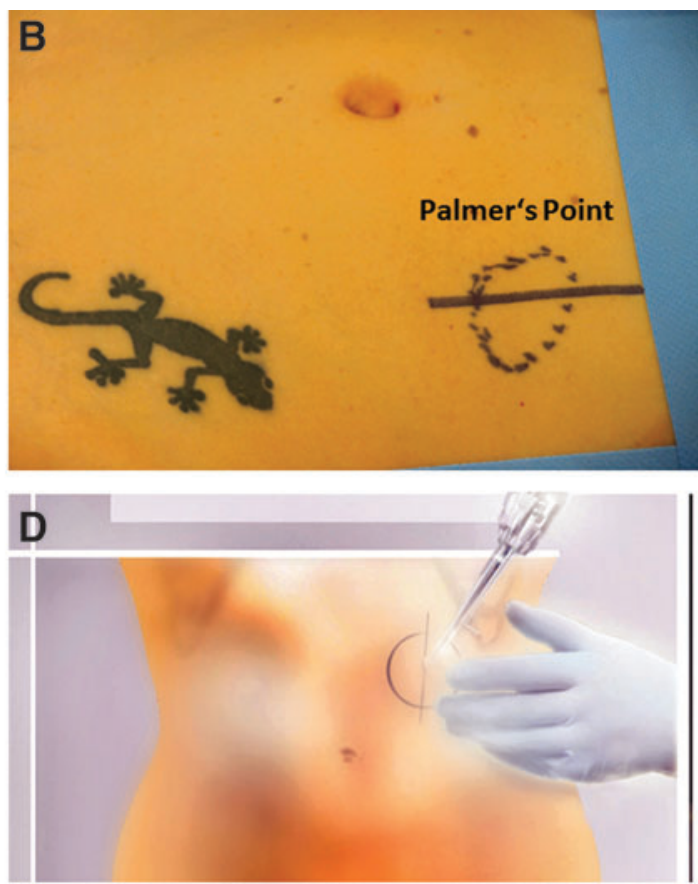

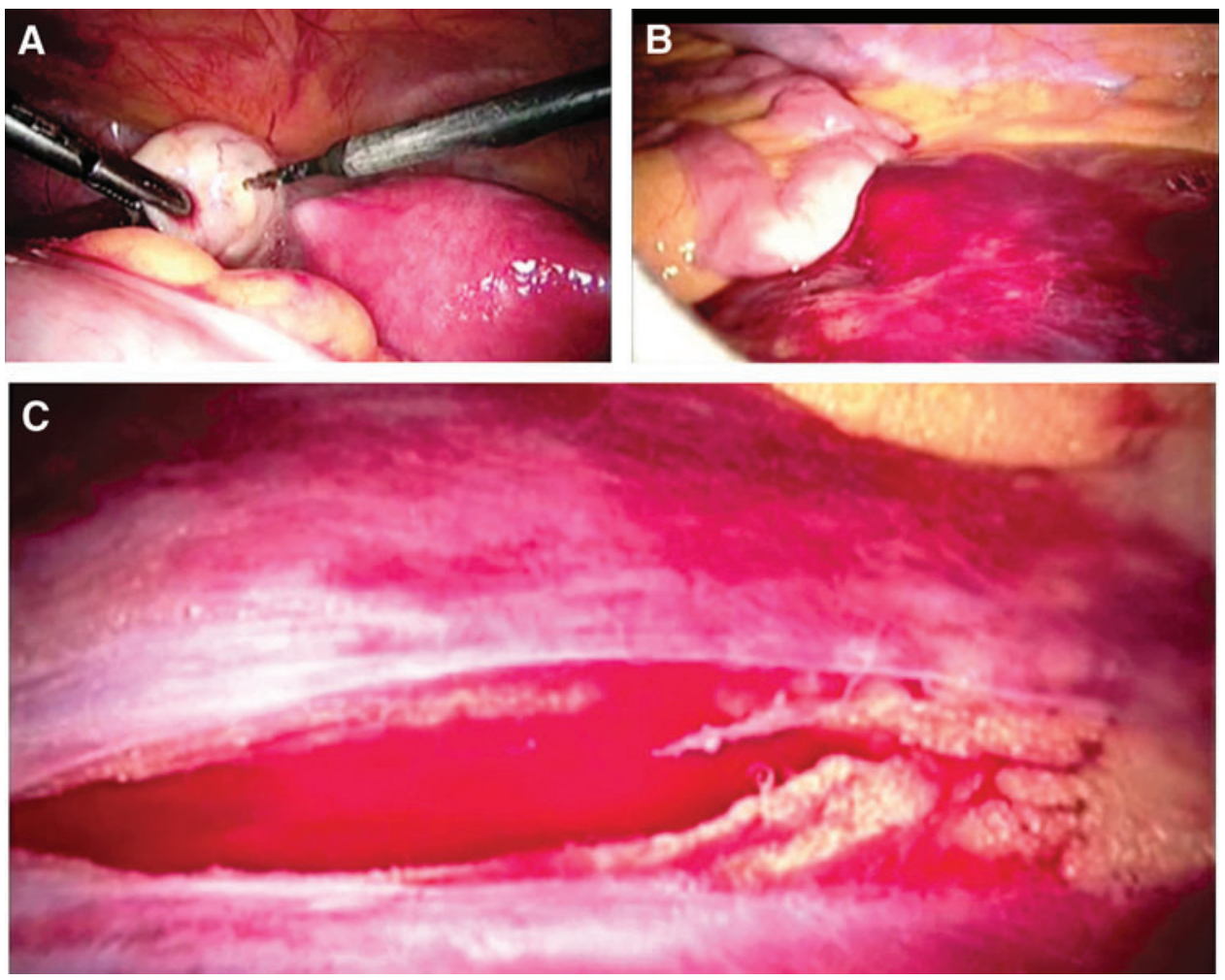

FIG. 5. (A) Monopolar drilling of the ovary just before ending the operation. (B) The final view reveals a massive retroperitoneal hematoma increasing in size. (C) Detailed inspection of the retroperitoneum shows a laceration just above the vena cava.
Laparoscopic entry lesions may be classified as follows:

- Type 1 injuries-Damage to major blood vessels or the bowel in a normal location, caused by entering with the Veress needle or the primary trocars $(0.1-0.4 \%)$

- Type 2 injuries-Damage to vessels in the abdominal wall and to the bowel adherent to the abdominal wall, both caused by the Veress needle or the pri- mary trocar; type 2 lesions may occur regardless of whether the mode of access is laparotomy or laparoscopy.

Complications associated with laparoscopy vary, depending on the experience of the surgeon and the medical staff as well as the wide range of operational demands. Complication rates vary between $0.1 \%$ and $1.3 \%$.
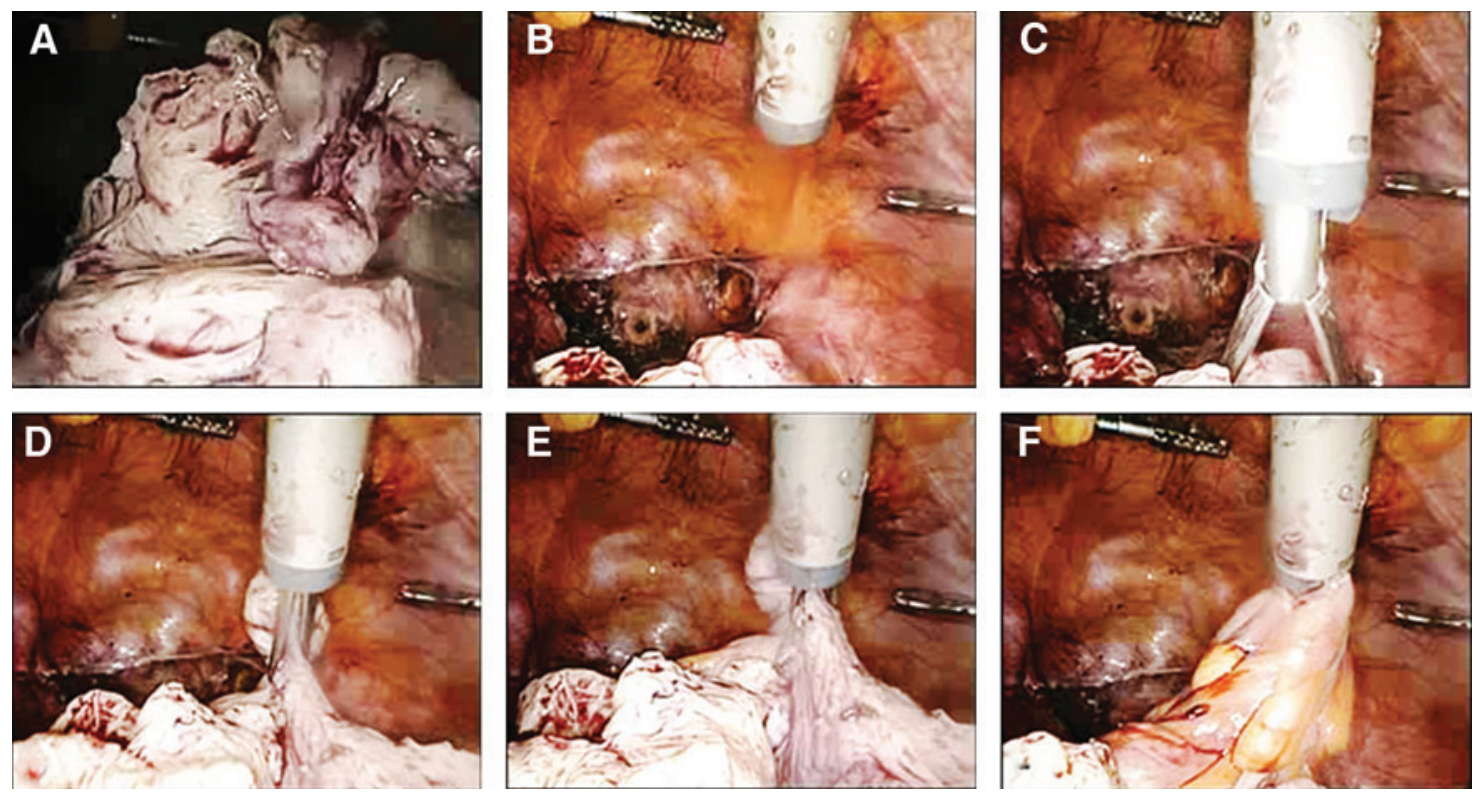

FIG. 6. (A) Morcellation of a medium-sized uterus. (B) The morcellator has been inserted into the midline; this ensures the best overview and the greatest distance to the organs. (C and D) Grasping the remaining uterus. (E) The tenaculum has grasped a small intestinal sling but, as the area of interest is not in the middle of the picture and the camera is too far away, the problem is not identified. (F) The surgical team identifies the bowel injury only after morcellation of the intestine. 
FIG. 7. A 32-year-old patient with continuous vaginal bleeding, para 1, with suspected placental remnants 5 weeks after delivery. (A and B) The soft anterior wall of the enlarged uterus was perforated during curettage for removal of placental remnants. The extent of perforation was identified during the simultaneous laparoscopy. Active bleeding, hematoma, and free fluid (rinsing fluid used for hysteroscopy). (C and D) The hole can be closed with a thick monofilament suture and intracorporeal knotting.
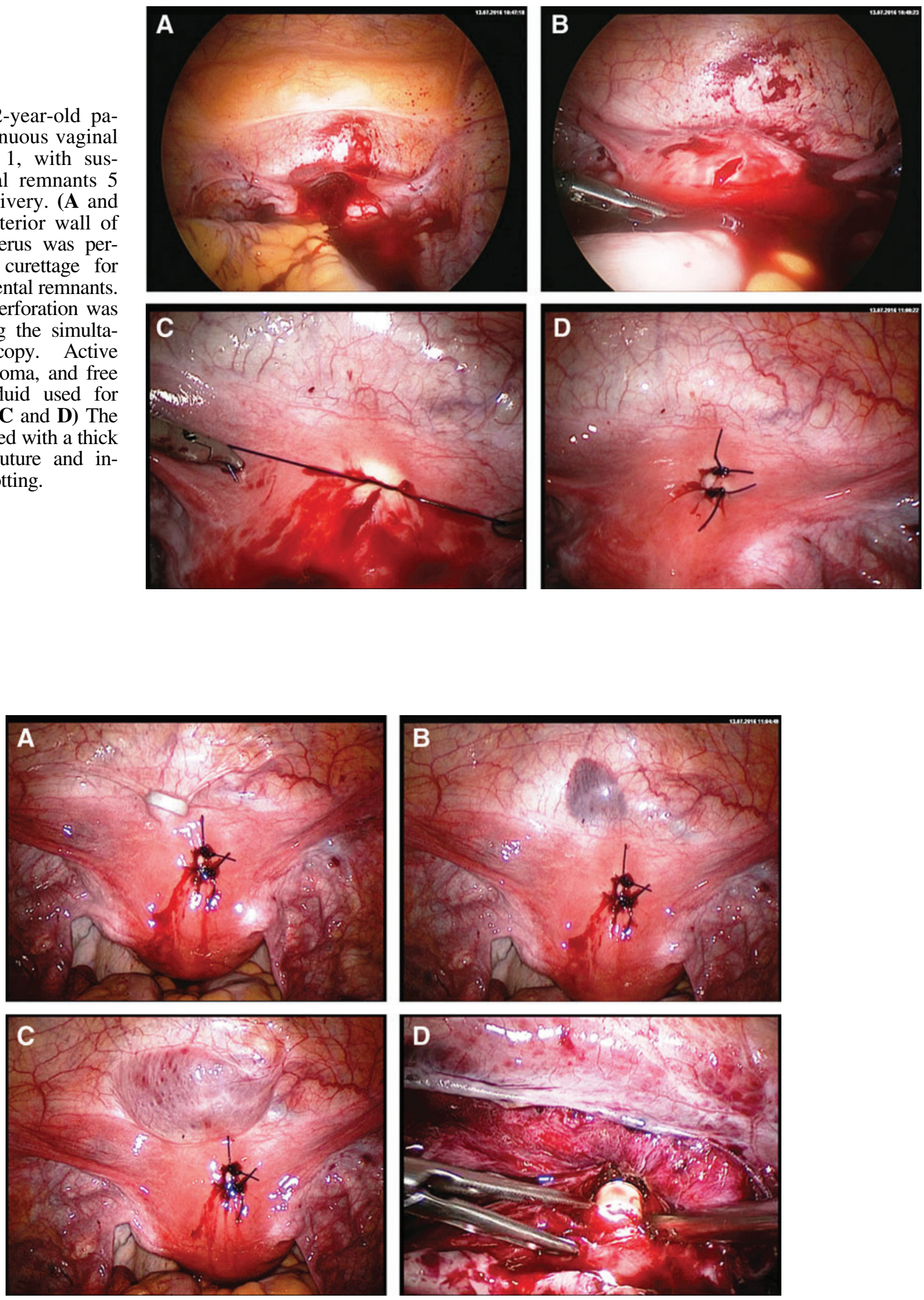

FIG. 8. The same patient as in Figure 7. (A) Attempt to perform curettage under laparoscopic vision reveals a second perforation at the cervico-corporal transition. Only the peritoneum of the bladder has remained intact. (B) Immediate subperitoneal bleeding. (C) An early attempt to manage the area of perforation by performing a hysteroscopy leads to a large edema in the subperitoneal space. (D) The peritoneum needs to be opened. After complete exposure of the anterior uterine wall down to the vagina, the sole hysteroscope is inserted without dilution medium, the perforation is demarcated and treated under laparoscopic vision. The left forceps is used to pull the distal part of the perforated tissue onto the distal part of the hysteroscope. The distal portion of the uterine corpus can now be examined. 

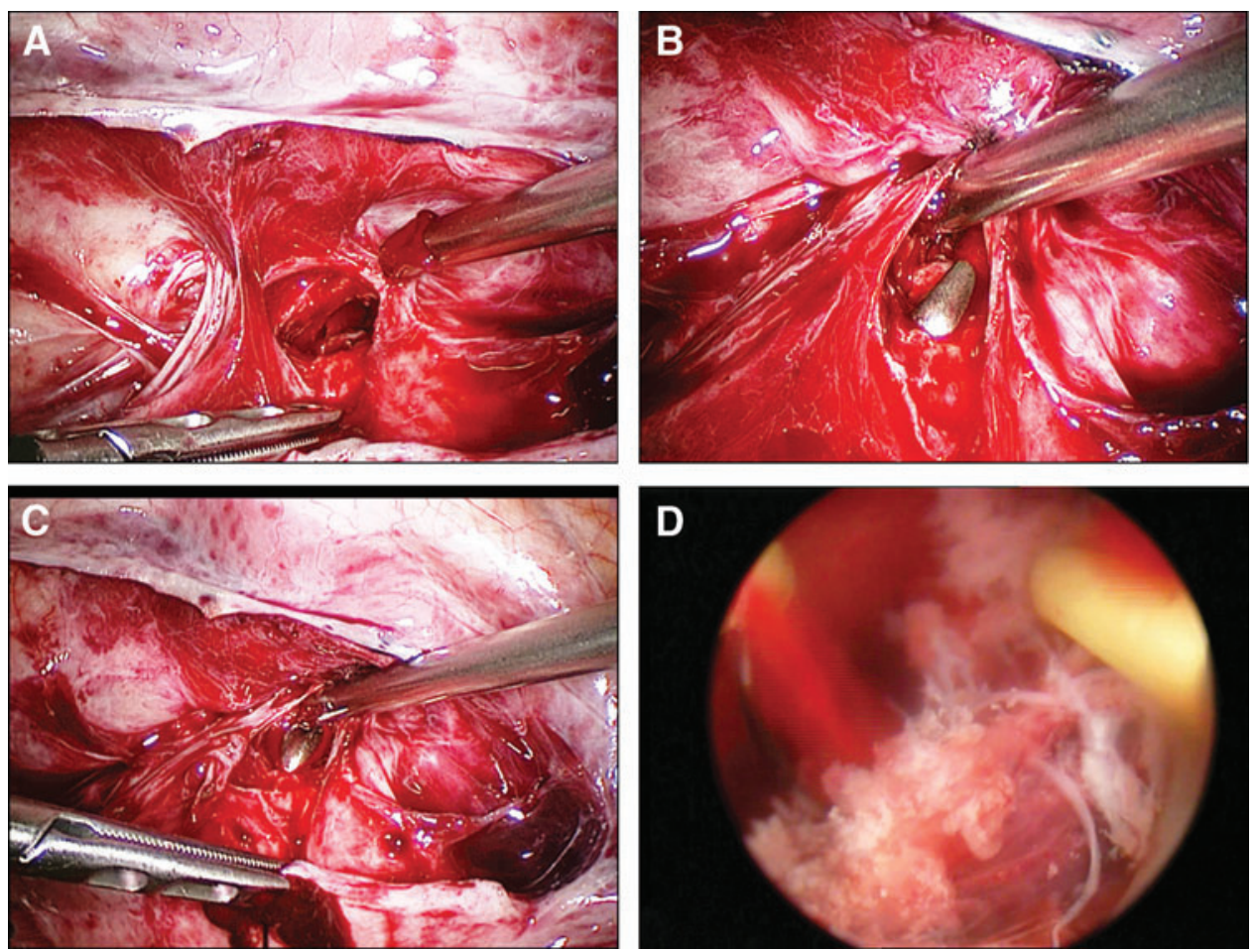

FIG. 9. The same patient as in Figures 7 and 8. (A) After complete exposure of the anterior uterine wall, only the vesicovaginal ligament is preserved. (B) A probe is inserted into the cervix and (C) directed into the uterine cavity under laparoscopic guidance. The defect is bridged by the probe and closed easily by making intracorporeal fullthickness sutures to the wall. (D) Hysteroscopic view of placental remnants, which may then be removed in a stepwise manner. After closure of the defect there is no further leakage and the dilution medium permits intrauterine vision.
The establishment of laparoscopic surgery for routine procedures at university teaching hospitals as well as at small county hospitals has enhanced the quality of the surgeon's work, which is now associated with a shorter learning curve. This advancement has been accompanied by a lower rate of complications. The development of laparoscopic instruments and optic transmission has also contributed to this phenomenon.
More than $50 \%$ of all laparoscopic injuries are known to occur during the initial entry steps: insertion of the Veress needle or blind entry; creation of a pneumoperitoneum; and insertion of the primary trocar. Accordingly, the incidence of injuries has been reduced significantly in the last 3 decades. Injury to the bowel occurs in $0.04 \%$ and to major vessels in $0.02 \%-0.04 \%$ of all laparoscopic procedures. Nevertheless, $30 \%-50 \%$ of bowel injuries and
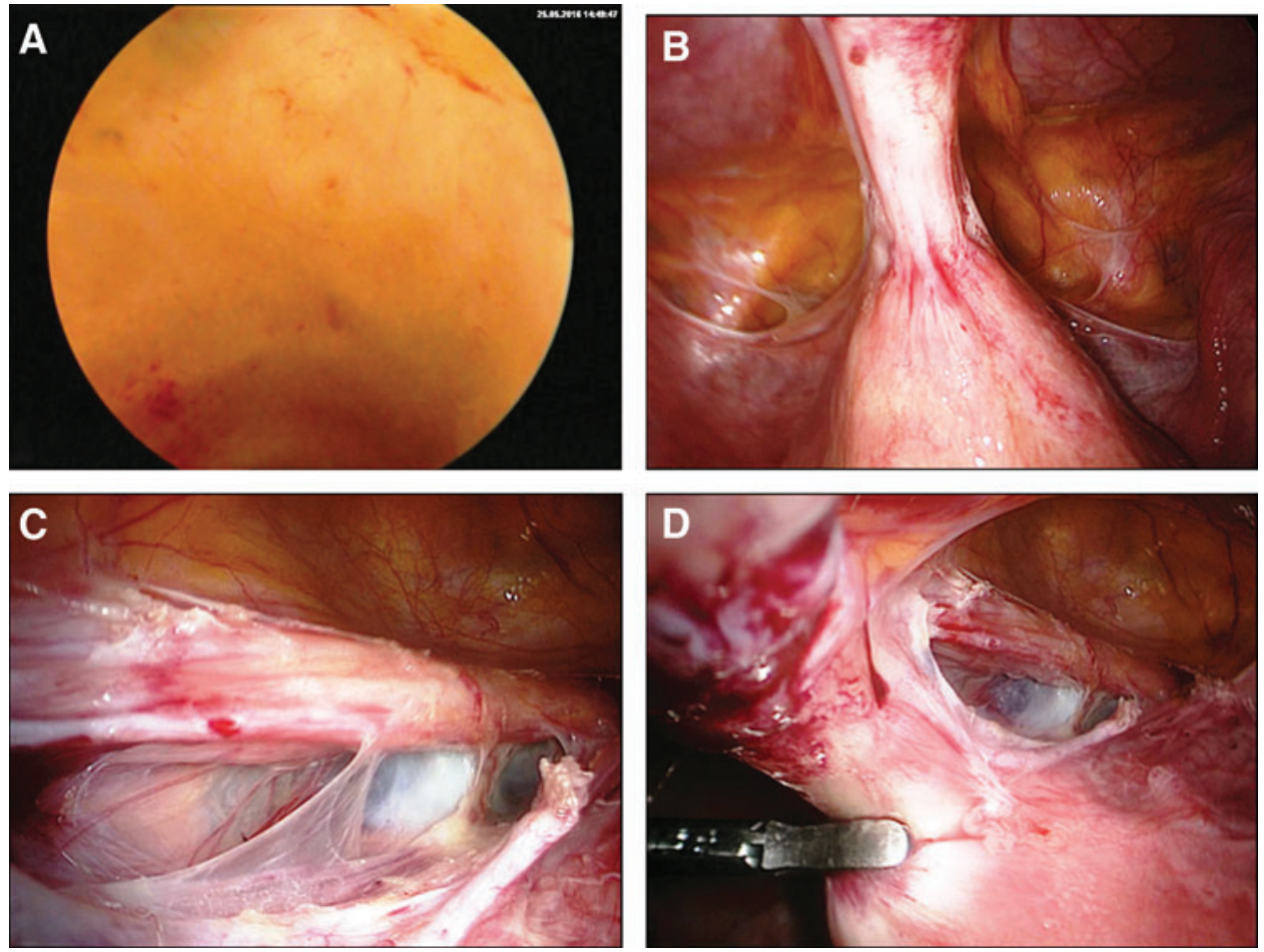

FIG. 10. A 35-year-old patient, para I, after cesarean section in a developing country (A), with a suspected defect of the cesarean scar. Hysteroscopy shows the margin of the intact wall; the defect seems to be covered by peritoneum alone. (B) The laparoscopic view shows a strong adhesive cord of the anterior uterine wall to the anterior abdominal wall. After opening the left (C) and the right (D) anterior leaf of the broad ligament, the bladder can be kept at a distance and the uterine defect demarcated. 
FIG. 11. Same patient as in Figure 10. (A) After complete adhesiolysis the bladder is moved away from the uterus and the deep defect demarcated. (B) A simultaneous hysteroscopy confirms that the defect covers the entire wall. (C) The thin cover of the defect is seen after the introduction of fluid through the hysteroscope. Additionally, the defect is demarcated by the illumination fixed to the hysteroscope. (D) Once the intra-abdominal illumination has been dimmed, the hysteroscopic illumination becomes very clear.
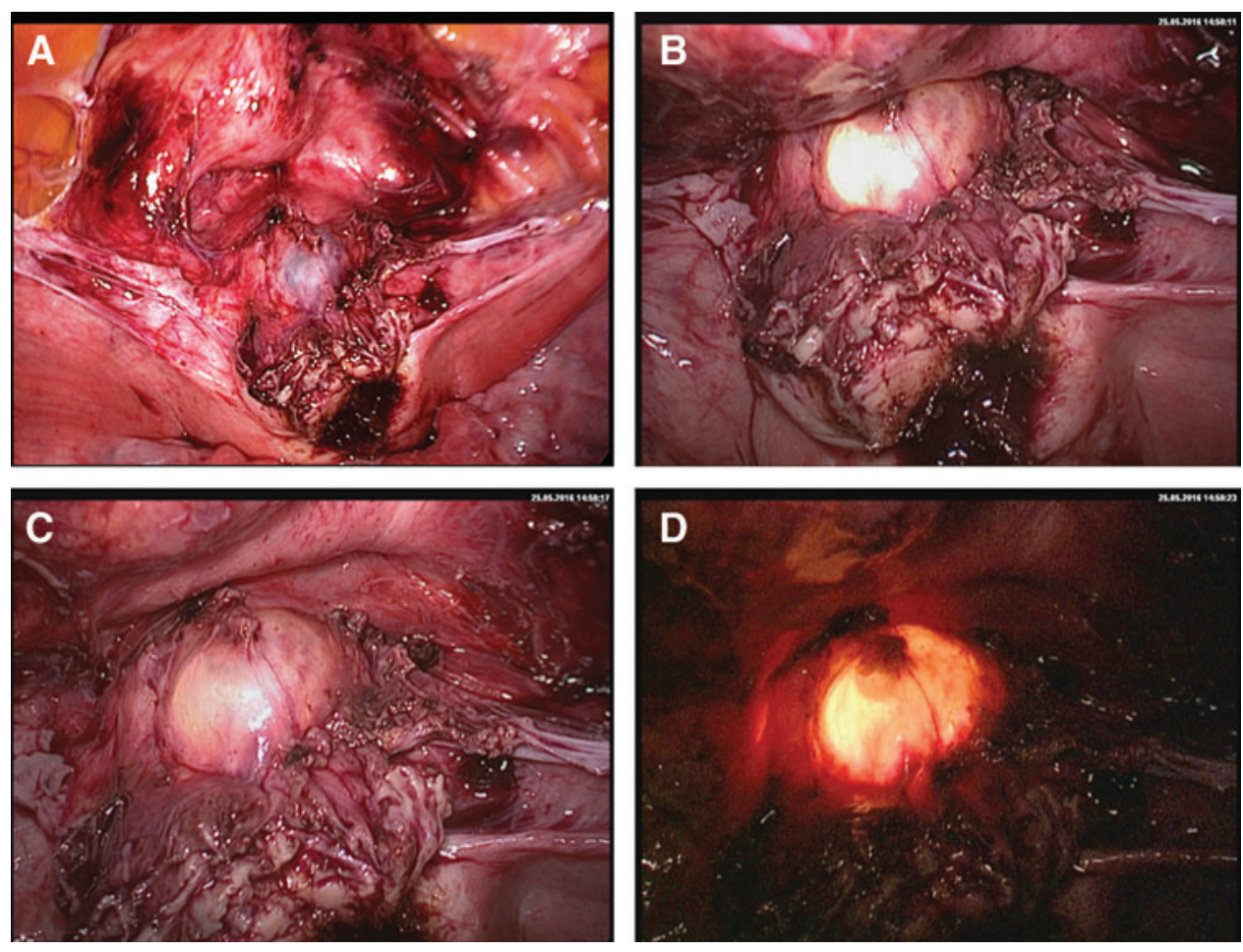

$13 \%-50 \%$ of all vascular injuries are not detected immediately during the operation, resulting in correspondingly high morbidity and mortality rates. Furthermore, laparoscopic surgery is frequently performed at outpatient clinics without banked human blood reserves, vascular surgical instruments, and expertise. Bowel injury ranks third among the causes of death from a laparoscopic procedure, being preceded by major vessel injury and anesthesia. $^{10-12}$

\section{Vascular and Visceral Lesions During Port Placement}

Vascular lesions may occur in the abdominal wall (superficial and epigastric vessels) or the intra-abdominal
FIG. 12. Same patient as in Figures 10 and 11. (A) Planned perforation with the hysteroscope allows (B) specific resection of the respective area. (C) The defect is demarcated and bridged with the hysteroscope (D), thus permitting endto-end coverage.
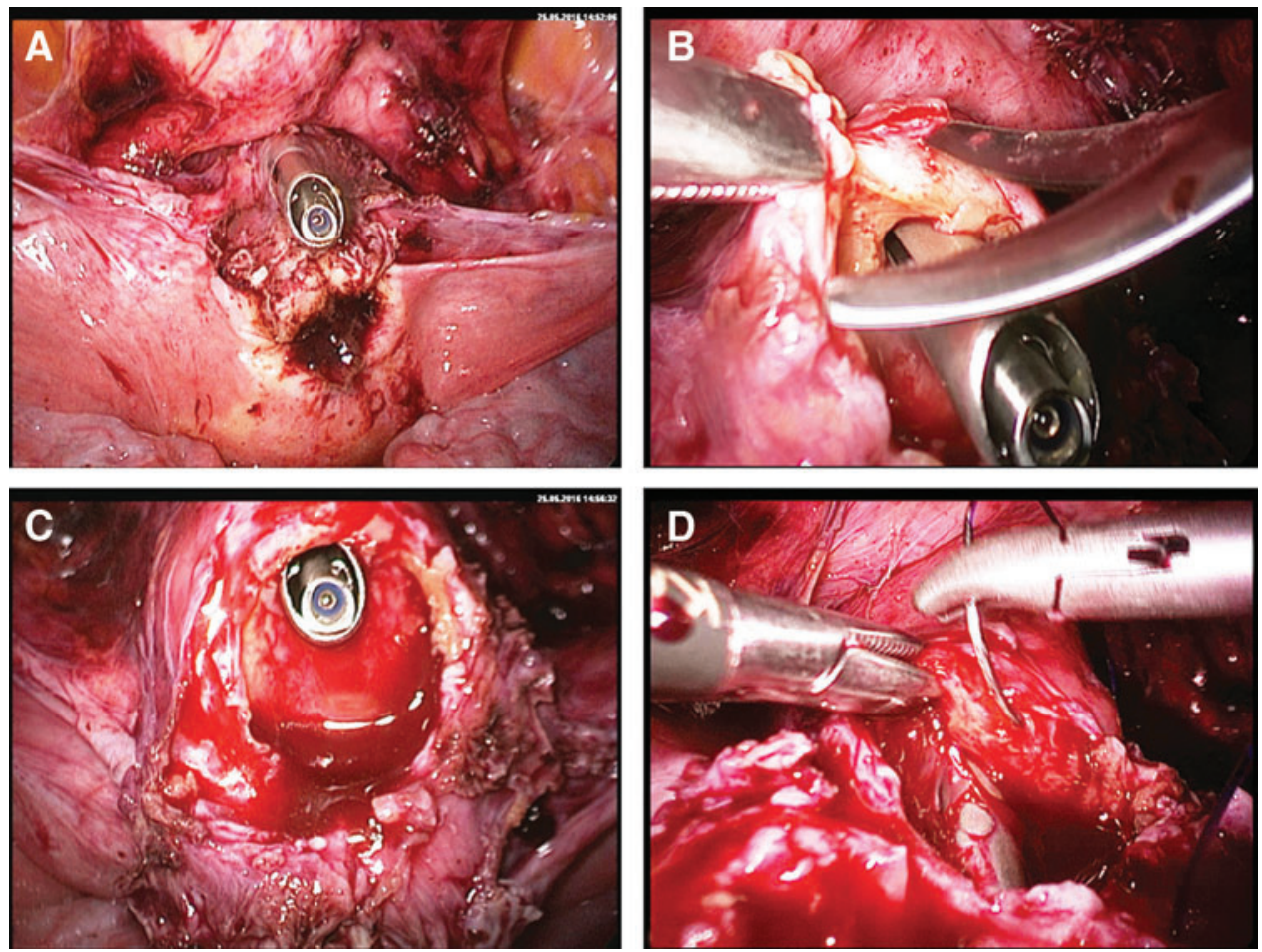

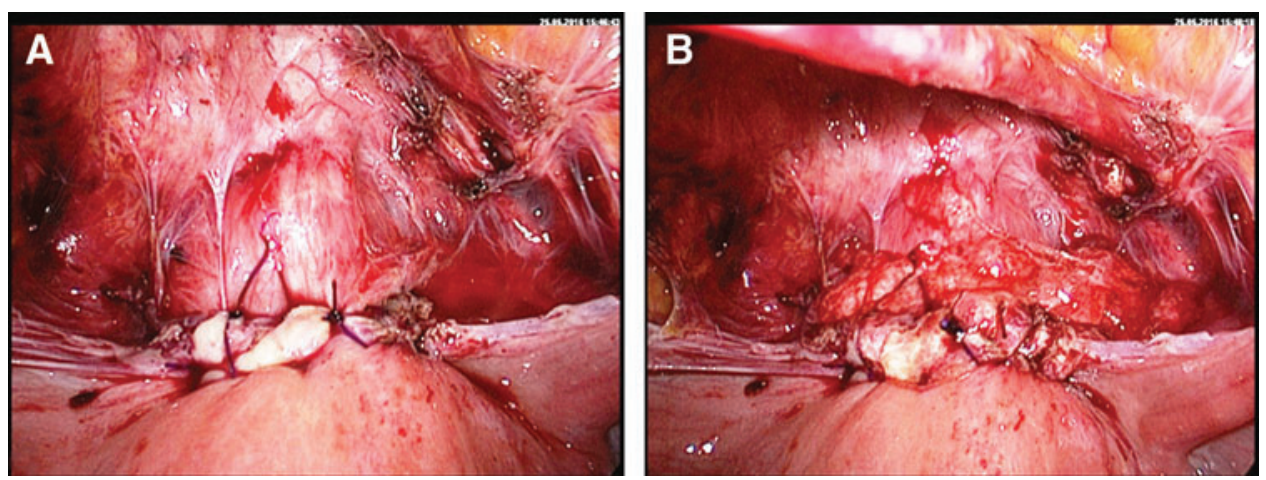

FIG. 13. Same patient as in Figures 10-12. (A) Final view of the covered defect of the anterior uterine wall. (B) As the patient still wishes to have children, an antiadhesion barrier gel is applied (Hyalobarrier, Nordic Pharma, Germany).

aspect (vessels of the mesentery, omentum, iliac arteries and veins, or the aorta/vena cava). Vessel injury may lead to a parietal hematoma or intraperitoneal hemorrhage, depending on the injury site (Figs. 3 and 5). Even partial entrance of the Veress needle into the lumen of a vessel may cause a gas embolism. In addition, $\mathrm{CO}_{2}$ is highly soluble in plasma. Large volumes can be lethal and are liable to cause immediate death. Injury to the retroperitoneal space and retroperitoneal structures can occur when using excessive force to insert the Veress needle, or even when using normal force in very slim patients; such injury may also occur when the surgeon fails to follow the midline axis during insertion of the primary trocar or employs an incorrect angle relative to the abdominal wall when entering the peritoneal cavity. As the aorta and the inferior vena cava bifurcate and diverge before entering the pelvis, it is important to minimize the risk of injury. ${ }^{7,13,14}$

\section{Visceral Lesions}

Visceral lesions include injury to the greater omentum, the stomach, the bowel, the liver, or the spleen, depending on the entry site; and the level of filling of the hollow organs. Therefore, a gastric tube must be placed at the beginning of the operation, especially when using Palmer's point or the Lee-Huang point.
As the first step in ensuring a safe procedure, penetration of the bowel can be identified by the aspiration of gas or the presence of ambiguous or malodorous fluid. Injury to the liver or the spleen would lead to the aspiration of blood. Depending on the members of the interdisciplinary team, injury to visceral organs can be treated laparoscopically or, if necessary, by immediate laparotomy (Figs. 1 \& 6). ${ }^{6,13,15-18}$

\section{Early Complications}

Early complications include bowel, vessel, bladder, or ureter lesions as well as anesthesia-related or general complications, such as pulmonary embolism, massive hemorrhage after major vessel injury or intravascular insufflation, and cardiac arrest.

Early complications may also occur in hysteroscopy (Figs. 7-9) and cannot be entirely avoided even by the most experienced surgeon when performing a laparoscopic procedure or a laparotomy (Figs. 10-13). The surgeon's expertise is manifested by speed and skill in identifying and dealing with complications.

\section{Vascular Lesions}

Major vessel injury may occur during the operative part of the procedure, particularly during retroperitoneal dissection. ${ }^{11}$ In the normal anatomical situation, the distal abdominal aorta and the common as well as external and
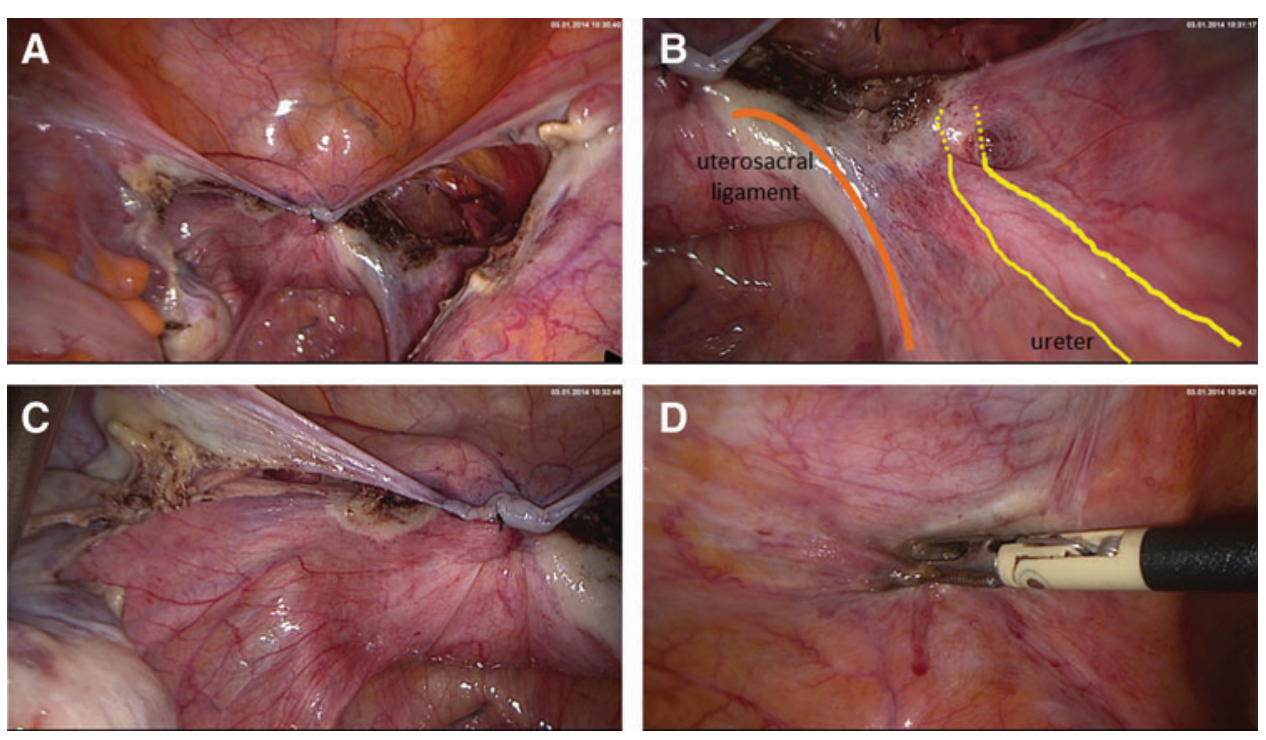

FIG. 14. Laparoscopic supracervical hysterectomy (LSH): Final view after the LSH procedure. The peritoneum closes the cervical canal and drainage is possible from both sides. Both sacrouterine ligaments are under slight tension and thus achieve suspension of the cervix. The preserved integrity of the ureter is confirmed by observing peristalsis (AC). Coagulation of the right trocar entry point after removal (D). 
FIG. 15. Opening the bladder usually requires the simultaneous insertion of a ureteral stent. This is achieved via cystoscopy (A and B). When the trigone of the bladder and the intramural pathway of the ureter are distant to the laceration, the latter can be closed securely by laparoscopy (C and $\mathbf{D})$. The hole is closed by making an interior suture line with Vicryl 3.0, using a continuous suture, and an interrupted suture line above with Vicryl 2.0 including the muscle and the peritoneum.
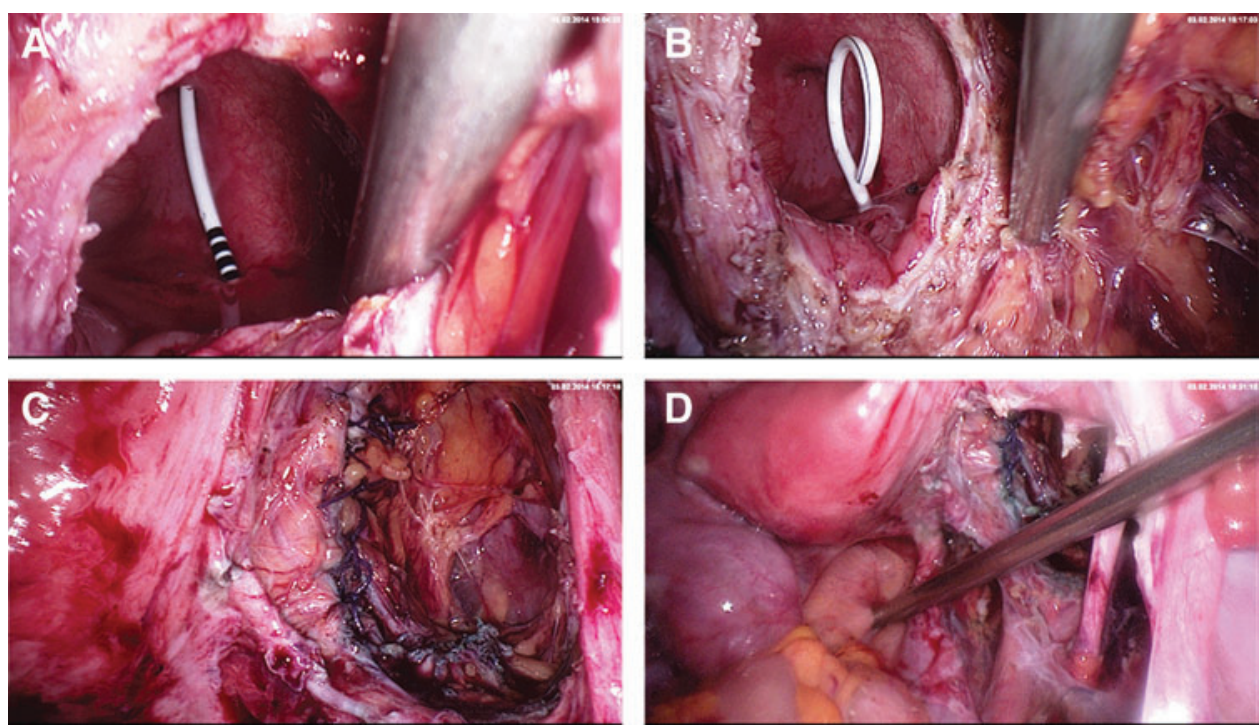

internal iliac arteries lie in the retroperitoneal space. Lacerations of these vessels are fortunately rare (Fig. 5).

Early recognition of a major vessel injury, minimization of bleeding, and conversion to laparotomy when the bleeding cannot be compressed laparoscopically, arrested, or sutured, are crucial aspects of effective management. Medical hemostasis might be helpful, such as the use of TachoSil. A vascular surgeon would be needed to conclude the operation.

\section{Bowel Lesions}

Abdominal access and the creation of a pneumoperitoneum bear a significant risk of bowel injury, which is more common in laparoscopic surgery than in open surgery. Although bowel injuries are uncommon, they constitute a major cause of mortality due to laparoscopic procedures and a significant source of morbidity in connection with any laparoscopic procedure.

Many intraoperative bowel lesions can be sutured; a partial excision and suturing as well as resection of lacerated areas could be necessary, including end-to-end anastomosis or a temporary ileostomy. Unlike major vessel injuries, which are seen immediately, many bowel injuries remain concealed at the time of the procedure. Patients may present postoperatively with specific or unspecific symptoms of peritonitis. Persistent pyrexia, tachycardia, or an ileus in the postoperative period should raise suspicion of bowel injury.
FIG. 16. (A) Second-look laparoscopy 3 days after nonproblematic myoma enucleation of the uterine back wall. The patient experienced bouts of high temperature and had increasing inflammation parameters. No drain was inserted. The overview shows normal pelvic anatomy and no sign of peritonitis or organ damage. (B) Atypical adhesions in the right lower abdomen (cecal region). (C) The endoscope clearly demarcates the bowel damage and signs of local peritonitis. The bowel injury probably occurred when the bowel was moved out of the operating field. (D) The upper right quadrant is also free of any signs of peritonitis.
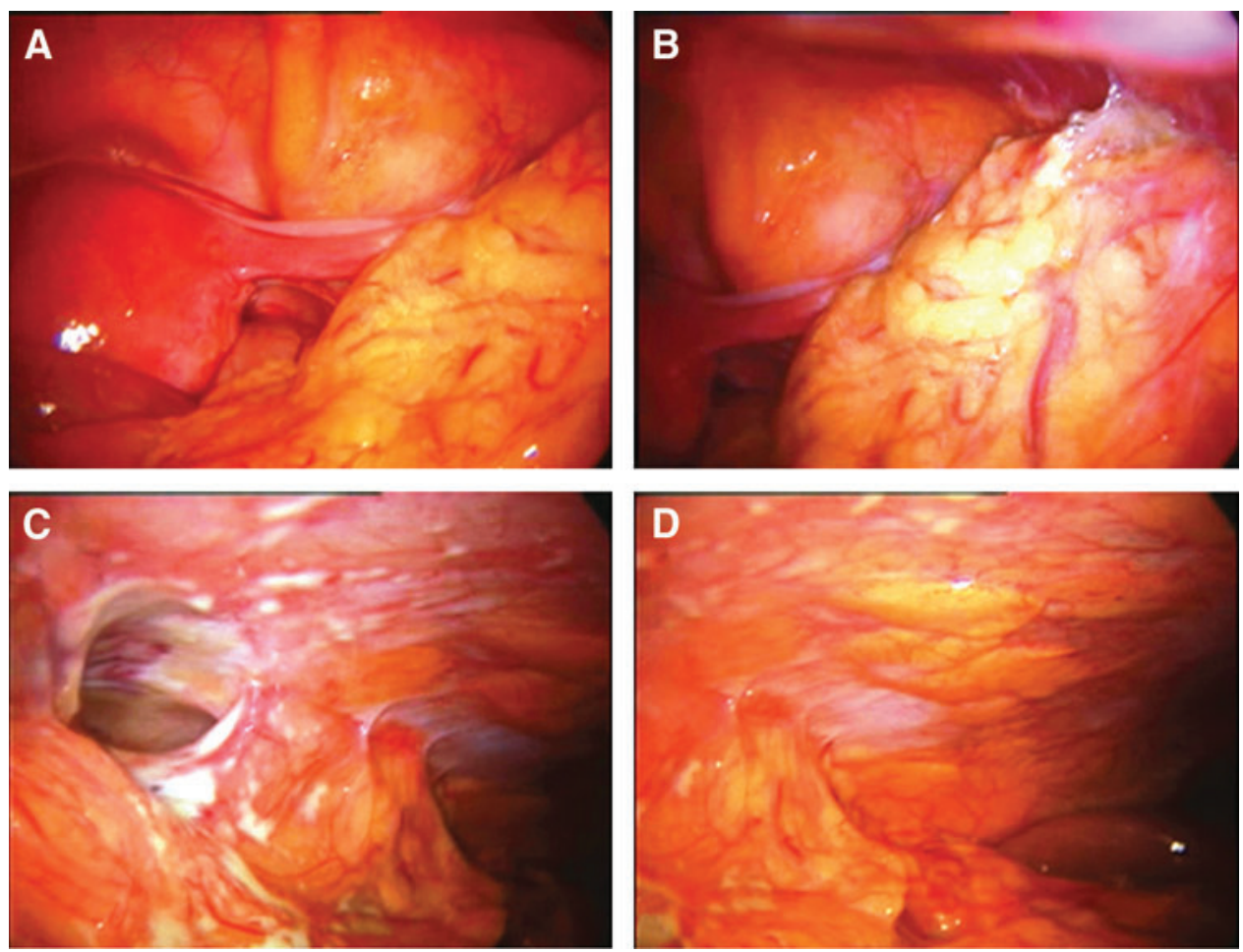
Late detection of this condition makes it a significant cause of morbidity and mortality. Bowel preparation is no longer advisable before major pelvic surgery. Injuries with healthy edges can be repaired primarily by using tension-free, singlelayer, interrupted sero-submucosal 3-0 Vicryl or 4-0 polydioxanone sutures. For more extensive injuries, resection and primary anastomoses may be required (Figs. 1 and 6).

\section{Bladder and Ureter Injuries}

Intraoperative viewing of the ureter is always necessary when performing surgery in the ureteric area. Dissection of the ureter becomes necessary sometimes (Fig. 14). Routine intraoperative cystoscopies after major gynecologic surgery permit early recognition and repair during the primary operation, with less morbidity for the patient. An open bladder is, of course, also detected by direct emission of urine. Sometimes the Foley catheter bag is filled with $\mathrm{CO}_{2}$, indicating a bladder lesion. ${ }^{19}$

Urinary-tract injuries in connection with laparoscopic surgery differ markedly from laparoscopic major vessel injury or iatrogenic intestinal injury. The former rarely results in the death of the patient, but the latter two are associated with mortality. Urinary complications are seldom the result of needle or trocar trauma (in other words, entry related).

The most relevant factors related to ureteral damage are: (A) inadequate knowledge of pelvic anatomy; (B) failure to open the peritoneum and dissect retroperitoneally; (C) the use of energy devices with marginal knowledge of their physics and tissue interaction; (D) imprecise application of stapling devices; and (E) pelvic adhesions, particularly dense adhesions located in and around the ovarian fossa.

Injury to the ureter is greatly compounded by its late identification after the operation. Failure to perform suitable diagnostic tests (such as indigo carmine dye injection, cystoscopy, intravenous pyelogram, or retrograde pyelograms) causes additional damage. Bladder injuries may be less serious than ureteral injuries, particularly when lacerations are recognized intraoperatively and repaired appropriately in a timely manner. Injury to the trigone may be avoided by performing a cystoscopy before or during closure of a bladder laceration and inserting a double-J-catheter (Fig. 15). The risk of injury, especially to the ureter, is higher when using the laparoscopic approach, particularly in laparoscopic gynecologic operations.

\section{Late Complications}

Late complications occurring in the postoperative period are also referred to as secondary lesions and occur in $0.5 \%$ of cases; these include secondary bowel lesions associated with peritonitis and massive intra-abdominal infection (Fig. 16). Small vascular lesions may remain unnoticed until a hematoma appears. Some ureteral lesions remain unidentified until the development of an urinoma; this may occur several days after surgery. All patients must be cautioned to immediately report adverse symptoms or conditions, regardless of whether they are still in the hospital or have been discharged.

\section{Conclusions and Future Perspectives}

Independent of the type of entry technique used, the surgeon must be well-trained in the technique of his/her preference. Furthermore, all surgeons should be familiar with alternative entry sites and techniques in order to resolve any type of obstacle or complication. The surgeon's regular attendance of standard training courses is known to shorten the learning curve.

Laparoscopic surgeons of all disciplines tend to divide laparoscopic surgery into various pillars, one of which is laparoscopic entry. During the training of new surgeons, attention should be given to laparoscopic entry alongside the more popular pillars of endoscopic exposure and suturing. Awareness of potential complications, their early detection, and professional treatment are essential aspects of a highquality operating unit. , 20,21 $^{2}$

All surgical procedures, including port placement, even under direct vision, are associated with immanent risks. ${ }^{22}$ Regardless of the sophisticated technological options of current times, doctors and surgeons need to work with great care and immense caution.

\section{Acknowledgments}

The current author thanks Mathias Podlovics for the graphic illustrations, Dawn Rüther, BA, for editing the manuscript, and Thoralf Schollmeyer, $\mathrm{MD}, \mathrm{PhD}$, for his valuable contributions.

\section{Author Disclosure Statement}

No competing financial interests exist.

\section{References}

1. Alkatout I, Bojahr B, Dittmann L, et al. Precarious preoperative diagnostics and hints for the laparoscopic excision of uterine adenomatoid tumors: Two exemplary cases and literature review. Fertil Steril 2011;95:1119.

2. Alkatout I, Stuhlmann-Laeisz C, Mettler L, Jonat W, Schollmeyer T. Organ-preserving management of ovarian pregnancies by laparoscopic approach. Fertil Steril 2011; 95:2467.

3. Royal College of Obstetricians and Gynaecologists (RCOG). Green-top Guideline No.49: Laparoscopic Injuries. London: RCOG. 2008:1.

4. Mettler L, Clevin L, Ternamian A, Puntambekar S, Schollmeyer T, Alkatout I. The past, present and future of minimally invasive endoscopy in gynecology: A review and speculative outlook. Minim Invasive Ther Allied Technol 2013;22:210.

5. Pasic R. Creation of pneumoperitoneum and trocar insertion techniques. In: Pasic R, Levine R, eds. A Practical Manual of Laparoscopy: A Clinical Cookbook. Abingdon, UK: Informa Healthcare, 2007:57.

6. Palmer R. Safety in laparoscopy. J Reprod Med 1974;13:1.

7. Nezhat F, Brill AI, Nezhat CH, Nezhat A, Seidman DS, Nezhat C. Laparoscopic appraisal of the anatomic relationship of the umbilicus to the aortic bifurcation. J Am Assoc Gynecol Laparosc 1998;5:135.

8. Alkatout I, Honemeyer U, Strauss A, et al. Clinical diagnosis and treatment of ectopic pregnancy. Obstet Gynecol Surv 2013;68:571. 
9. Alkatout I, Schollmeyer T, Hawaldar NA, Sharma N, Mettler L. Principles and safety measures of electrosurgery in laparoscopy. JSLS 2012;16:130.

10. Vilos GA, Vilos AG, Abu-Rafea B, Hollett-Caines J, Nikkhah-Abyaneh Z, Edris F. Three simple steps during closed laparoscopic entry may minimize major injuries. Surg Endosc 2009;23:758.

11. Vilos GA, Ternamian A, Dempster J, Laberge PY; Society of Obstetricians and Gynaecologists of Canada. Laparoscopic entry: A review of techniques, technologies, and complications [in English \& French]. J Obstet Gynaecol Can 2007;29:433.

12. Magrina JF. Complications of laparoscopic surgery. Clin Obstet Gynecol 2002;45:469.

13. Hurd WW, Bude RO, DeLancey JO, Pearl ML. The relationship of the umbilicus to the aortic bifurcation: Implications for laparoscopic technique. Obstet Gynecol 1992; 80:48.

14. Hurd WH, Bude RO, DeLancey JO, Gauvin JM, Aisen AM. Abdominal wall characterization with magnetic resonance imaging and computed tomography: The effect of obesity on the laparoscopic approach. J Reprod Med 1991;36:473.

15. Semm K. New methods of pelviscopy (gynecologic laparoscopy) for myomectomy, ovariectomy, tubectomy and adnectomy. Endoscopy 1979;11:85.

16. Semm K. Visible control of peritoneal perforation in surgical pelviscopy [in German]. Geburtshilfe Frauenheilkd 1988;48:436.

17. Semm K. Morcellement and suturing using pelviscopy-not a problem any more [in German]. Geburtshilfe Frauenheilkd 1991;51:843.
18. Semm K. Cutting versus conical tip designs. Endosc Surg Allied Technol 1995;3:39.

19. Mettler L, Schollmeyer T, Tinelli A, Malvasi A, Alkatout I. Complications of uterine fibroids and their management, surgical management of fibroids, laparoscopy and hysteroscopy versus hysterectomy, haemorrhage, adhesions, and complications. Obstet Gynecol Int 2012;2012: 791248.

20. Alkatout I, Mettler L, Beteta C, et al. Combined surgical and hormone therapy for endometriosis is the most effective treatment: Prospective, randomized, controlled trial. J Minim Invasive Gynecol 2013;20:473.

21. Mettler L, Sammur W, Schollmeyer T, Alkatout I. Crosslinked sodium hyaluronate, an anti-adhesion barrier gel in gynaecological endoscopic surgery. Minim Invasive Ther Allied Technol 2013;22:260.

22. Mettler L, Schollmeyer T, Alkatout I. Adhesions during and after surgical procedures, their prevention and impact on women's health. Womens Health (Lond) 2012;8:495.

Address correspondence to: Ibrahim Alkatout, $M D, P h D, M A$ Department of Gynecology and Obstetrics Kiel School of Gynecological Endoscopy University Hospitals Schleswig-Holstein

Campus Kiel Arnold-Heller Straße 3, House 24 24105 Kiel Germany

E-mail: kiel.school@uksh.de 